\title{
BAJO EL SIGNO DE LAS DIFERENCIAS: UNA APROXIMACIÓN EDUSEMIÓTICA A LA VULNERABILIDAD
}

Under the Sign of Differences: An Edusemiotic Approach to Vulnerability

Susana Gómez-Redondo, Universidad de Valladolid, España

Correo electrónico: susana.gomezr@uva.es

Francisco José Francisco Carrera, Universidad de Valladolid, España Correo electrónico: franciscojose.francisco@uva.es

Lidia Sanz Molina, Universidad de Valladolid, España

Correo electrónico: lidia.sanz@soc.uva.es

Recibido: $15 / 05 / 2021$

Aceptado: $15 / 11 / 2021$

Publicado: 07/01/2022

Resumen. Desde la consideración de que el ser humano es "signo entre signos" y ser relacional, estas páginas suponen una aproximación a las conexiones existentes entre la edusemiótica (especialmente en su dimensión ética), y las concepciones en torno a las diferencias y la vulnerabilidad humana. A partir de la semiótica de Peirce y la edusemiótica propuesta por Semetsky, contempla postulados de la ética del cuidado de Nodding y perspectivas socioculturales y hermenéuticas.

Palabras clave: edusemiótica, educación, signo, semiosis, diferencias, vulnerabilidad.

Abstract. From the consideration that the human being is a sign among other signs and a relational being, the following pages offer an approach to the existing connections between edusemiotics (especially in its ethical dimension) and the conceptions around the differences and human vulnerability. Based on Peirce's semiotics and the edusemiotic proposal by Semetsky, it comtemplates the principles Nodding's ethics of care as well as sociocultural and hermenetical perspectives.

Keywords: edusemiotics, education, sign, semiosis, differences, vulnerability.

Cómo citar: Gómez-Redondo, S., Francisco Carrera, F. J., y Sanz Molina, L. (2022). Bajo el signo de las diferencias: una aproximación edusemiótica a la vulnerabilidad. EN-CLAVES del pensamiento, 0(31), e495. doi: https://doi.org/10.46530/ecdp.v0i31.495 
¿Cómo imagino la relación maestro-alumno? Por lo pronto, como una relación en la que el maestro no pretende poseer el monopolio de la Verdad... Desde el momento en que habitamos el mundo vivimos no solamente con los otros, sino frente a ellos [...] Este otro singular que me apela o me encara me recuerda que nunca estoy completamente solo en la configuración del sentido, en la invención del sentido (o sentidos). Esta presencia del otro me conmina a decidir éticamente [...] somos éticos porque desde el comienzo estamos en un tiempo y en un espacio en-relación- con-los-otros. Somos seres relacionales.

Joan-Carles Mèlich

La edusemiótica puede ser descrita como el campo de conocimiento que resulta de la unión entre la semiótica, o estudio de los signos, y la teoría y/o la filosofía de la educación. Se trata, pues, de una rama de la teoría semiótica y de la filosofía aplicada, que estudia el proceso de significación de los signos en el contexto educativo. Fue presentada formalmente como disciplina autónoma en el marco del XII Congreso Mundial de la Asociación Internacional para Estudios Semióticos (IASS por sus siglas en inglés), celebrado en 2014 en la New Bulgarian University de Sofía. Cuatro años antes, un volumen colectivo había recogido bajo el título de Semiotics Education Experience ${ }^{1}$ diversas aportaciones sobre las confluencias entre la educación y la semiótica. En el prólogo, a cargo del editor jefe de la prestigiosa revista Semiótica, Marcel Danesi, se vaticinaba que dicho trabajo bien podría constituir el texto fundacional de una "verdadera edusemiótica para el futuro". 2

La incipiente disciplina venía, así, a sumarse a otras ramas de la semiótica teórica, como la biosemiótica. No obstante, aquel texto seminal en el que se enunciaran algunas de las fundamentaciones teóricas y epistemológicas de un campo aún por inaugurar oficialmente, no era el primer trabajo que advertía de la pertinencia de un dialogo entre educación y semiótica. Ya en 1973, Kondrátov, biólogo, poeta y uno de los lingüistas más representativos de Rusia, exponía la necesidad de una interacción entre ambas áreas: "La semiótica es necesaria para la pedagogía, pues todo el proceso del aprendizaje se reduce a que los hombres aprendan a manejar los signos, ya sea los de la lengua materna o extranjera, ya sea los signos matemáticos, los de la física, de la química o de otras ciencias". ${ }^{3}$

\footnotetext{
${ }^{1}$ Inna Semetsky (Ed.), Semiotics Education Experience (Rotterdam: Sense Publishers, 2010).

2 Marcel Danesi, "Foreword: Edusemiotics", en Inna Semetsky (ed.), Semiotics Education Experience (Rotterdam: Sense Publishers, 2010). La traducción es nuestra.

${ }^{3}$ Aleksandr Mikhaĭlovic Kondrátov, Del sonido al signo (Buenos Aires: Paidós, 1973), 31.
} 
Por su parte, y como también remarcara Danesi en su prólogo introductorio, Vigotsky había destacado con anterioridad que un aspecto esencial de la memoria humana es que recurrimos a los signos para recordar. Creemos que Danesi sintetiza acertadamente la cuestión edusemiótica que subyace en este planteamiento, al defender que en el mismo se puede encontrar la 'razón de ser' de establecer una conexión entre la semiótica (como ciencia de los signos); la teoría del aprendizaje, o ciencia que describe cómo los estudiantes reciben, procesan y retienen el conocimiento (o lo que es lo mismo, cómo los signos son aprendidos) ${ }^{4}$ y la educación (entendida esta como arte o ciencia destinada a enseñar a la persona a interpretar y entender los signos). ${ }^{5}$

Figura 1. Relación entre semiótica, teoría del aprendizaje y educación

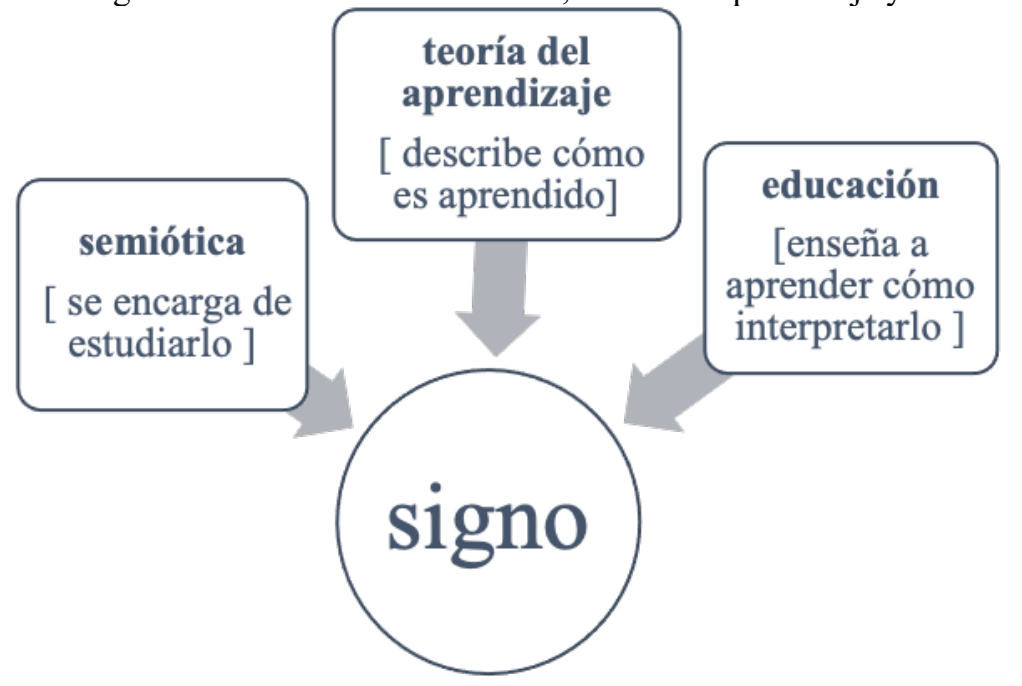

Fuente: elaboración propia a partir de Danesi /Vigotsky.

En sus estudios en torno a la interacción entre pensamiento y lenguaje, Vigotsky llevaría a cabo reiteradas incursiones en los "campos lindantes" de la lingüística y la psicología de la educación ${ }^{6}$. Tales investigaciones contribuyeron a esclarecer, por vez primera de un modo sistemático y desde propuestas experimentales, las conexiones que ponen de relieve la estrecha relación entre palabra y cognición. No es raro, pues, encontrar entre los postulados teóricos del padre de la psicología histórico-cultural determinados vórtices, en los que se dejan ver algunas de las líneas de fuerza de la futura edusemiótica.

\footnotetext{
${ }^{4}$ Este ámbito guarda relación con los esfuerzos actuales de la semiótica por determinar el lugar que ocupa el estudio de los procesos y estructuras mentales, en la comprensión de los procesos de construcción de sentido. ${ }^{5}$ Marcel Danesi, "Foreword: Edusemiotics", en Semetsky (ed.), Semiotics Education Experience.

${ }^{6}$ Lev Semionovich Vigotsky, Pensamiento y lenguaje (Buenos Aires: Fausto, 1995).
} 


\section{La semiosis como creatividad, novedad y conectividad}

Frente a la concepción semiológica de Saussure, que restringía el signo a las expresiones lingüísticas y verbales, la semiótica propuesta por Charles Sanders Peirce abrirá su foco hasta llegar a abarcar a toda la existencia. Es así, desde una visión "pansemiótica y naturalista", 7 como el fundador del pragmatismo y la semiótica moderna formula una teoría general del signo y la representación, la cual incluye toda manifestación, ya sea humana o no humana.

Peirce supera la propuesta dualista de Saussure (quien divide el signo en significante y significado), fundamentando la relación sígnica en una estructura triádica irreductible. Tal concepción permite comprender al conocimiento (y, por tanto, su evolución y la adquisición de nuevos conocimientos a partir de otros anteriores) como un proceso de significación en el que siempre intervienen tres elementos: signo, objeto e interpretante:

Un signo, o representamen, es algo que está por algo para alguien en algún aspecto o capacidad. Se dirige a alguien, esto es, crea en la mente de esa persona un signo equivalente o, tal vez, un signo más desarrollado. Aquel signo que crea lo llamo interpretante del primer signo. El signo está por algo: su objeto. Está por ese objeto no en todos los aspectos, sino en referencia a una especie de idea, a la que a veces he llamado fundamento [ground] del representamen. ${ }^{8}$

A este proceso, o acción del signo, Peirce lo denominó “semiosis". En él, podemos considerar que la permanente incompletud de la acción sígnica (inacabamiento que posibilita el cambio y la adquisición de nuevos significados) arroja un enfoque permanentemente creativo y dinámico: un signo da lugar a otro, gracias a un proceso transformativo, continuo e ilimitado, en el que la interacción es su fuerza motriz. La dimensión relacional es, pues, inherente al proceso de generación de nuevos significados.

Al igual que lo hiciera la ciencia de la interpretación, al dejar atrás la hermenéutica del texto para dar paso a la hermenéutica de la existencia formulada por Heidegger, ${ }^{9}$ la

\footnotetext{
7 Inna Semetsky, "Introduction: A Primer on Edusemiotics", en Inna Semetsky (Ed.) Edusemiotics. A Handbook (Singapore: Springer, 2017).

${ }^{8}$ Charles S. Peirce, Fundamento, objeto e interpretante (2.228, c. 1897). Texto tomado de MS 798 [On Signs] c.1897, 5. Fue publicado como Collected Papers (CP) 2.227-229 y 2.444n1. Trad. Mariluz Restrepo, 2003.

9 En el primer tercio del siglo XIX, Schleiermaher, máximo exponente de la hermenéutica romántica, defendería que el arte de la interpretación no debía limitarse únicamente a las producciones literarias, sino que
} 
semiótica de Peirce supera la restringida visión del signo lingüístico y afirma que todo es signo. Toda expresión, toda experiencia cumple, por tanto, las características esenciales del signo. Esto es: es susceptible de ser interpretada; tiene capacidad de mediar entre el objeto y el interpretante y puede llevar una idea a la mente de dicho interpretante. Semetsky se refiere a este proceso de la siguiente manera:

Semiosis es un proceso comunicativo, interactivo, relacional e interpretativo. La comunicación, que es el flujo de información y la mutua transformación de los signos que son transformados en otros signos, es un importante concepto en semiótica [...] Sin embargo, los signos no son solamente producidos intencionadamente con el propósito de comunicar, como en la semiología; [...] la acción de los signos se manifiesta también en síntomas, sueños y el inconsciente en psicoanálisis. ${ }^{10}$

Podemos decir, entonces, que la semiosis es: omnímoda, en el sentido de que abraza y abarca todo (esto incluye tanto los signos de la naturaleza como los de la cultura y la mente humana, ya sean lingüísticos o extralingüísticos); dinámica, porque enfatiza los procesos de crecimiento y modificación de los signos a través de la acción, la transformación y la evolución; ilimitada y creativa, pues un signo da lugar a otro, en un proceso de creación y (re)creación que nunca termina debido a su radical y permanente incompletud. Y, por último, es esencialmente relacional, en tanto en cuanto el signo creado siempre tiene que ver con los anteriores a él, del mismo modo que se conectará con los subsiguientes a los que dé lugar.

Desde esta visión omnímoda o pansemiótica, los pensamientos (igual que los aprendizajes y el conocimiento) son signos. De ahí que la lógica, en sentido amplio, no sea para Peirce "sino otro nombre para la semiótica, la doctrina cuasi-necesaria, o formal, de los signos". ${ }^{11}$ Como procesos dinámicos de acción, creación y evolución, los pensamientossigno son interpretados, desarrollados o modificados (esto es, confirmados, desechados o implementados), dando lugar a otro/s, transformado/s y distinto/s de los anteriores y subsiguientes. Esta constante reelaboración y resignificación, que pone en conexión el pasado y el futuro, responde a la ya apuntada característica de infinitud, generatividad e

se había de extender a todo fenómeno comprensivo (Friedrich Schleiermaher, Hermeneutics and criticism and other writings. Cambridge: Cambridge University Press, 1989). Finalmente, sería Heidegger el responsable de equipararla definitivamente con la misma existencia. Ello supuso el paso de una hermenéutica de los textos a una hermenéutica de la existencia, según la cual la comprensión y la interpretación son procesos fundamentales que hallamos en el corazón de la vida misma. Desde esta perspectiva, todo es texto, en tanto en cuanto todo puede ser leído e interpretado. Toda experiencia puede ser, por tanto, objeto de la hermenéutica.

${ }^{10}$ Inna Semetsky, "Introduction: A Primer on Edusemiotics", in Semetsky (Ed.) Edusemiotics. A Handbook, 3.

${ }^{11}$ Charles S. Peirce. (CP 2.227, c. 1897), in Collected Papers vol 1-8, C. Hartshorne, P. Weiss y A. W. Burks (Eds.) (Cambridge, MA: Harvard University Press, 1931-1958) 
interacción. El proceso, lejos de ser automático, necesita de la imaginación y la inteligencia para definir, hacer crecer y clarificar progresivamente (interpretar y comprender, en terminología hermenéutica) los signos que participan en la acción semiótica. Pero crecer, para Peirce, no es solo aumentar, sino dar lugar a nuevos signos que son distintos a sí (su objeto o referente), con lo que esto implica de novedad y, diríamos, salir de sí. La semiosis como la educación, es, pues, creatividad, innovación, conectividad y encuentro.

En términos hermenéuticos, el círculo o la espiral representarían gráficamente no solo el crecimiento y la novedad, sino la profundidad comprensiva y, por tanto, la construcción de conocimiento y aprendizaje. En cuanto a este salir de sí en busca del otro, del análogo, será la hermenéutica analógica de Beuchot ${ }^{12}$ la que insistirá más claramente en esa visión intersubjetiva y de exploración de la otredad.

En su clásica comparación con la célula como unidad de análisis biológico, Vigotsky definió el significado como la unidad más pequeña de pensamiento y lenguaje. El psicólogo encontraría en esta unidad de significado muchas de las respuestas a la interrelación entre palabra-signo y cognición.

En esta misma línea, y si nos atenemos a los fundamentos de la teoría celular, la unidad "palabra-significado" vendrá a ser unidad morfológica y funcional de todo pensamiento, necesariamente derivada de otros significados precedentes y potencial generadora de otros nuevos. Como las células y el signo semiótico, dicha unidad estará sujeta a transformaciones e interconexiones, responsables de la creación de otros signos (palabras y conocimientos), los cuales requerirán de la inteligencia y la imaginación. Del mismo modo, los procesos de desarrollo tendrán lugar gracias a unidades cognitivas, socioafectivas y, derivadas de la dimensión sociocultural del ser humano, éticas y morales.

En consonancia con los enfoques sociohistóricos y las teorías marxistas, toda unidad se verá entonces fuertemente afectada por un contexto, el cual influye poderosamente en la evolución sígnica (en este caso, el desarrollo cognitivo-afectivo del niño). No hay que olvidar que, para Vigotsky, ${ }^{13}$ el entorno juega, por encima de las creencias y actitudes, un papel esencial en el qué y el cómo se piensa. Frente a las teorías innatistas, el medio es un factor determinante en la construcción de la persona.

\footnotetext{
12 Mauricio Beuchot. Tratado de hermenéutica analógica: hacia un nuevo modelo de interpretación (Ciudad de México: Universidad Autónoma Metropolitana, 2000); Mauricio Beuchot. Hermenéutica, analogía y símbolo (Barcelona: Herder, 2004); Mauricio Beuchot, Perfiles esenciales de la hermenéutica (Ciudad de México: Fondo de Cultura Económica, 2008).

${ }^{13}$ Lev Semionovich Vigotsky, Pensamiento y lenguaje (Barcelona: Paidós Ibérica, 2020).
} 
Por su parte, Piaget $^{14}$ propone el aprendizaje como una reorganización de las estructuras cognitivas que se producen en cada momento. Esta idea supone entender los cambios cognoscitivos como procesos en construcción, en los que la propia experiencia (los postulados constructivistas apuntan al individuo como el principal hacedor de su aprendizaje) junto con el medio modifican los conocimientos, aportando unos nuevos. De este modo, y desde perspectivas semióticas, el desarrollo intelectual puede ser visto como un proceso de reestructuración del conocimiento-signo que, influido por un cambio externo, modificará la estructura existente, dando lugar a nuevas unidades de signos-conocimiento. Como resultado de la interacción entre los factores cognitivos y sociales, dicho proceso tendrá lugar en cualquier entorno en el que la persona realice sus interacciones.

Siguiendo con esta apropiación de las teorías socio-culturales, el significado (como proceso o acto de significar) se modificará entonces en función de la realidad contextual ( agma $^{15}$ o flujo sociocultural en donde signo, objeto e interpretante flotan y se hacen posibles). En este sentido, apuntamos que el contexto, en su función activa de mediación (y no de simple medio amniótico o placentario), podría ser considerado un cuarto factor a sumar a la triada sígnica, en tanto en cuanto tiene el poder de intervenir sobre sus tres elementos constitutivos. Tal visión iría en consonancia con las teorías hermenéuticas que, en otra clásica triada (texto-contexto-autor), confieren al contexto un papel esencial en el proceso hermenéutico-comunicativo.

La edusemiótica se sitúa claramente en un marco conceptual integrador. No solo porque es el fruto directo de una simbiosis disciplinar, sino porque tiene como finalidad superar el dualismo cartesiano y su legado. Como entidad triádica, el signo semiótico remite a algo diferente a sí mismo (su objeto o referente) gracias a esa tercera categoría, el interpretante, cuya intervención rompe con la antigua dicotomía entre sujeto y objeto: en términos cartesianos, el carácter inmaterial de la res cogitans (realidad psíquica caracterizada por la inextensión, la libertad y la conciencia) y la res extensa (naturaleza material, física, extendida, limitada e inconsciente).

14 Jean Piaget, Introducción a la psicolingüistica (Buenos Aires: Nueva Visión, 1969); Jean Piaget, El lenguaje y el pensamiento del niño pequeño (Barcelona: Paidós, 1984); Jean Piaget, Inteligencia y afectividad. (Buenos Aires: Aique, 2001); Jean Piaget, El nacimiento de la inteligencia en el niño (Barcelona: Editorial Crítica, 2014).

${ }^{15}$ Cornelius Castoriadis, La institución imaginaria de la sociedad (Barcelona: Tusquets, 2013). 
Como resume Semetsky, la disciplina edusemiótica se nutre de presupuestos ontológicos, entre cuyos precursores filosóficos se incluyen, además de Peirce, pensadores como Platón, Leibniz, James, Dewey o Whitehead. Junto a ellos se pueden encontrar algunos de los pioneros de las filosofías hermética y neoplatónica, así como representantes de las corrientes filosóficas orientales. De este modo, la edusemiótica no solo continúa, sino que reinterpreta, en los nuevos y actuales contextos, el legado de los principales filósofos y críticos teóricos (desde el pragmatismo americano hasta la tradición continental), y revisita filosofías tan ancestrales como el Hermetismo o el Taoísmo. ${ }^{16}$

Entre los puentes hermenéuticos insistimos en vindicar especialmente a Mauricio Beuchot, cuya vocación de cruce propone un espacio de encuentro, analógico, prudente y no dicotómico, entre objeto y sujeto, modernidad y posmodernidad, univocidad y equivocidad, positivismo y relativismo..., por poner algunos ejemplos antiéticos que la concepción sintética hermenéutico-analógica pone a dialogar.

Además, la edusemiótica implica teorías y metodologías como la fenomenología, reafirmado los vínculos ya existentes en la apropiación que hiciera la semiótica de las teorías de Husserl, conquista que cuenta con el mérito de conciliar las tensiones entre el fervor por el texto de los estructuralistas y la devoción por el signo y la lógica de Peirce.

Todo lo anterior nos lleva a incidir en ese carácter integrador que defiende la superación de dicotomías, muy persistentes en buena parte del pensamiento occidental, como cuerpo-mente, sujeto-objeto positivismo-relativismo, verdad-error, razón-intuición, etc. Aspectos como la paradoja, la complejidad o la lógica del tercero incluido son, de este modo, muy afines a las propuestas edusemióticas, cuyos fundamentos suponen un desafío para el principio de la no contradicción. Semetsky las sintetiza del siguiente modo:

La edusemiótica no destaca lo verdadero frente lo falso o las respuestas correctas frente a las incorrectas como las oposiciones binarias que los profesores emplean normalmente para evaluar a sus estudiantes; lo que es importante es el proceso participativo de aprendizaje, en el que los estudiantes encuentran significación y sentido, y los profesores son responsables de crear un ambiente lo bastante participativo, en lugar de limitar la educación a su mero producto, que generalmente se limita a resultados determinados por test estandarizados. ${ }^{17}$

\footnotetext{
${ }^{16}$ Semetsky, "Introduction: A Primer on Edusemiotics", en Semetsky, Edusemiotics. A Handbook.

${ }^{17}$ Ibid., 7.
} 
Todo lo anterior cuestiona inercias educativas basadas en la dualidad y la homogenización del alumnado, como el sistema de calificaciones fundamentado en el logro o el fracaso; la ausencia sistemática y la penalización del pensamiento divergente frente al ensalzamiento del convergente, o las inercias transmisivas y la repetición como garantía de éxito.

\section{Dimensión relacional y ética del cuidado}

En cuanto sujeto semiótico, el ser humano está siempre sujeto a la posibilidad de crecimiento. ${ }^{18}$ Es en esta posibilidad e incompletud, fuerza motriz de los procesos educativos y razón de ser de su vocación de perfeccionamiento, ${ }^{19}$ donde reside a un tiempo su vulnerabilidad y fortaleza. Sin inacabamiento no hay necesidad de mejora, como tampoco hay menester del otro (los demás) ni de lo otro (el mundo que nos circunda). Signos entre signos, los seres humanos apelamos a la novedad, la creatividad y la conectividad para crecer y dar lugar a nuevos signos. Biológicamente menos determinados que el resto de los animales, acudimos a nuestra segunda naturaleza, la piel socio-cultural, para contextualizanos y desarrollar procesos pedagógicos, encaminados a la supervivencia y la optimización.

En la tarea de enseñar y aprender se pone en juego un rasgo básico de nuestro diseño cognitivo: "la capacidad de saber lo que sabemos y, por tanto, también lo que ignoramos; pero también de imaginar lo que otros saben y, por tanto, también lo que ignoran". ${ }^{20}$ Así entendida, la dimensión pedagógica sería, pues, específicamente humana: el Homo sapiens sapiens (el humano que sabe que sabe) no solo tiene, además, la necesidad de enseñar lo que sabe, sino que la satisface gracias a su habilidad para intuir y representarse lo que el otro necesita saber.

\footnotetext{
${ }^{18}$ Sara Barrena y Jaime Nubiola, Charles S. Peirce (1839-1914): Un pensador para el siglo XXI (Navarra: Ediciones Universidad de Navarra, 2013).

19 Ya desde la antigua Grecia, la concepción predominante sobre la educación (tanto en su asociación etimológica más tradicional, el vocablo educare, como en su concepción más moderna, la acepción educere) es la de perfeccionamiento. Tal idea presupone que la educación se dirige a mejorar al ser humano, gracias a procesos de transformación destinados a la optimización de la persona, es decir, a que pase de un estado a otro mejor.

${ }^{20}$ Juan Ignacio Pozo, "La nueva cultura del aprendizaje en la sociedad del conocimiento", en Pozo et al. (Eds.), Nuevas formas de pensar la enseñanza y el aprendizaje: las concepciones de profesores y alumnos (Barcelona: Graó, 2015), 35.
} 
Esta capacidad metarrepresentacional es fundamento del proceso educativo y en ella radica la esencia del homo pedagógico, pues denota nuestra disposición, diferenciada y única, para elaborar representaciones y signos internos que no solo expliquen, sino que además predigan, al otro. La pedagogía será la sistematización de esta capacidad que, en un diálogo entre presupuestos internalistas y externalistas, racionales y poéticos, diseñará y pondrá en marcha acciones socioeducativas, a partir de esa triple dimensión de la didáctica técnica, normativa y creativa.

El Homo discens ${ }^{21}$ u homo pedagógico es, pues, testaferro y deudor de procesos socioeducativos (signos en permanente evolución) que se transmitirán, modificarán y mejorarán gracias a la acción pedagógica. Este proceso o acción educativa requiere de la capacidad humana de salir de sí, pues solo en el encuentro con el otro y con lo otro puede darse.

Este sería, en suma, el corazón del hecho socioeducativo. El ser humano, como signo entres signos en un contexto socio-cultural determinado, sobrevive y evoluciona gracias a un delicado equilibrio entre la perpetuación de lo ya conocido (los signos precedentes) y su natural propensión a innovar (la búsqueda de nuevos signos). Impulsados por nuestra configuración gnoseológica, tenderemos a constantes puentes entre las estructuras racionales y relacionales del individuo y la comunidad: la terna interactiva entre el yo, lo otro y el otro.

Será este vínculo con los demás lo que nos humanice. Dicho de otro modo: nacemos humanos, pero nos humanizamos gracias al contacto, en el entorno, con aquellos que nos rodean. Para Vigotsky, como para Marx, lo esencialmente humano nace de las relaciones sociales. Como signos entre signos, seremos relacionales o no seremos.

Es esta dimensión relacional del signo en la que hemos venido insistiendo la que nos interesa especialmente, pues creemos, con Savater, que la educación es el momento de la ética. ${ }^{22}$ La semiosis educativa, como proceso comunicativo, interactivo, relacional e interpretativo (en propuesta de Semetsky) y omnímodo, dinámico, ilimitado, creativo y

\footnotetext{
${ }^{21}$ Juan Ignacio Pozo, Adquisición del conocimiento (Madrid: Morata, 2003).

22 Fernando Savater, "La educación es el momento adecuado de la ética. ¿De qué sirve la ética para los jóvenes?”, Educere, 4, núm. 11 (2000): 215-226. Conferencia celebrada en la Universidad Simón Bolívar de Caracas, Venezuela, con motivo de nombramiento de Doctor Honoris Causa otorgado el 29 de octubre de 1999.
} 
relacional (en la nuestra) se fundamenta en ese diálogo con uno mismo, con los demás y el mundo. En esta triada relacional reside nuestro crecimiento y, también, los conflictos y tensiones derivados de los intereses, libertades y límites del individuo frente a la colectividad.

En su propuesta mesoaxiológica de la educación, Touriñán afirma los siguiente:

Se conjuga el cultivo de lo personal y la grandeza de miras, porque la educación, entendida en su sentido pleno, no alcanza su objetivo con desarrollar un hombre capaz de valerse por sí mismo y para sí mismo. Además, debe entenderse que este núcleo personal no estará conseguido mientras que la educación no salvaguarde y cultive en cada educando el sentido de la relación con el otro y lo otro, es decir el sentido social de la diversidad y la identidad en los espacios de convivencia, lo cual implica llegar a asumir el compromiso con los principales derechos y obligaciones que como ciudadanos y como miembros de la comunidad deben cumplirse en el marco legal territorializado. ${ }^{23}$

Del mismo modo que el signo perciano no se fagocita en sí mismo, sino que a través del interpretante se convierte en otro, la educación es, por definición, relación y alteridad. En un sentido, pues, similar a la de la Hermenéutica analógica, la visión edusemiótica encuentra en el otro y su interacción la verdadera medida del uno, estableciendo una geografía de derechos y responsabilidades entre el yo y los demás.

Entendemos que esta dimensión relacional y ética de la edusemiótica, que introduce aspectos como la diversidad y la identidad, entronca poderosamente con la visión de Noddings y su ética del cuidado, como perspectiva de una educación moral alternativa a la educación del carácter. Frente a esta última, fundamentada en la enseñanza de las virtudes aristotélicas, Noddings propone la relación de cuidado como punto de partida y elemento vertebrador de la educación moral. Su propuesta no se basa entonces en acciones preceptivas de dudosa eficacia (que muchos han equiparado al adoctrinamiento), sino en la creación de condiciones que fomenten la bondad como eje y motor del cuidado. De este modo, en lugar de la enseñanza directa de lo que es considerado 'bueno' (la pregunta es: ¿bueno para quién? y, en consecuencia: ¿los valores de quién?), este enfoque se basa en enseñar y aprender a cuidar para, a partir de ahí, construir los pilares de una educación moral no preceptiva, sino eminentemente relacional.

\footnotetext{
${ }^{23}$ José Manuel Touriñán, "Donde hay educación, hay riesgo: además de enseñar, hay que educar", Voces de la Educación, 1, núm. 1 (2016): 115.
} 
Esta idea confiere al contexto un papel fundamental en, al menos, dos aspectos: de un lado, lo propone como marco para la configuración de un medio, el socioeducativo, en el que el niño sea cuidado. Solo así estará amparada su vulnerabilidad y, además, aprenderá a cuidar. De otro, atiende al entorno (físico, social, emocional, etc.), como espacio contextualizador de valores éticos y morales en el que las virtudes habrán de ser reflexionadas y priorizadas en función de su evaluación. Nos gustaría señalar, al respecto, que consideramos que tal propuesta no cae en el relativismo, dado que parte de la necesidad de que el niño tenga una formación moral sólida, cuya integralidad e integridad logra trascender las fórmulas preceptivas. De este modo, la vulnerabilidad (la incompletud) bien puede ser entendida como referente de un proceso sígnico y educativo, que cree en el interpretante nuevas significaciones y sentidos, y dé lugar a nuevas unidades de conocimiento socioafectivo y ético.

Los vínculos entre los postulados de la edusemiótica y la propuesta de Noddings se evidencian en el hecho de que esta se centra más en la relación de cuidado que en el cuidado como virtud. Por ende, el "uso relacional ocupa un primer lugar". Podríamos decir que el signo sale de sí, para ponerse en relación con otro.

La ética del cuidado, que tiene sus raíces en el feminismo y en el naturalismo pragmático, supera además la dicotomía entre razón y sentimiento, cuando distingue entre cuidado natural y cuidado ético. En su perspectiva, el primero estará basado en el sentimiento natural, en tanto que el segundo se fundamentará en el refuerzo y la valorización del sentimiento hacia el propio yo ético.

Así mismo, la perspectiva feminista se traduce en Noddings en que el cuidado, indefectiblemente relacional, pone énfasis en la interacción y no en el agente. Esta vindicación del encuentro lima la tradicional asimetría entre persona que cuida y persona que es cuidada, estableciendo una reciprocidad en la interacción:

A diferencia de otras formas de ética, la teoría del cuidado reconoce en quien recibe cuidados un aporte especial, que no consiste en una respuesta recíproca de prodigar cuidados. Los niños hacen un aporte significativo a la relación madre-hijo; los alumnos a la relación docente-alumno, y los pacientes, a la relación médico-paciente. ${ }^{24}$

\footnotetext{
${ }^{24}$ Nel Noddings, La educación moral. Propuesta alternativa para la educación del carácter (Buenos Aires: Amorrortu, 2009), 21.
} 
En dicha interacción, Van Manen ${ }^{25}$ no confiere al adulto la verdadera autoridad pedagógica, sino que se la otorga al niño. Para el pedagogo, el adulto sensible a la vulnerabilidad infantil experimenta una exigencia ineludible: la pedagógica, convirtiéndose de este modo debilidad del niño en fuerza sobre el adulto. Van Manen atribuirá este intercambio a la "responsabilidad" para con el niño, valor que Arendt, en su pedagogía de la natalidad, hallará en el compromiso ético con los nacen o están por nacer y, en última instancia, con el amor por el mundo:

La Educación es el punto en el cual decidimos si amamos al mundo lo suficiente como para asumir una responsabilidad por él, y de esa manera salvarlo de la ruina inevitable que sobrevendría si no apareciera lo nuevo, lo joven. Y la Educación también es donde decidimos si amamos a nuestros niños lo suficiente como para no expulsarlos de nuestro mundo y dejarlos librados a sus propios recursos, ni robarles de las manos la posibilidad de llevar a cabo algo nuevo, algo que nosotros no previmos, si los amamos lo suficiente para prepararlos por adelantado para la tarea de renovar un mundo común. ${ }^{26}$

\section{Vulnerabilidad y diferencia}

La vulnerabilidad, entendida como "potencia de la fragilidad",27 apela a un adulto que no solo sea capaz de comprender, sino de sostener a otro, en el sentido otorgado por Ruddik de "minimizar los riesgos y conciliar las diferencias, en lugar de acentuarlas". ${ }^{28}$ Entendemos que tal acomodación se medirá en términos relacionales y no homogeneizadores, pues el principio de normalidad pedagógico corre el riesgo de la estandarización

En un sentido próximo, el pragmatismo de Dewey y sus teorías de pedagogía progresista se evidencian en Noddings en postulados relacionados con el contexto escolar y el respeto por la diversidad. Dewey consideraba que, de forma indiscriminada, obligamos a niños con distintas inclinaciones a realizar los mismos ejercicios, instalándolos en una “aburrida uniformidad". En un párrafo traspasado de amargura, escribiría en 1916: “después de malgastar nuestros esfuerzos en atrofiar los verdaderos dones de la naturaleza,

\footnotetext{
${ }^{25}$ Max Van Manen, El tacto en la enseñanza. El significado de la sensibilidad pedagógica (Barcelona: Paidós Educador, 2018).

${ }^{26}$ Hanna Arendt, "La crisis de la educación", en Entre el pasado y el futuro. Ocho ejercicios sobre la reflexión política, trad. de Ana Poljak (Barcelona: Ediciones Península, 1996, 185-208), 208.

27 Asun Pié Balaguer, La insurrección de la vunerabilidad. Para una pedagogía de los cuidados y la resistencia (Barcelona: Edicions Universitat Barcelona, 2019).

${ }^{28}$ Sara Ruddick, Maternal thinking: towards a politics of peace (Boston: Beacon Press, 1989), en Noddings, La educación moral, 61.
} 
vemos que el resplandor efímero e ilusorio con que los sustituimos desaparece, y que las habilidades naturales que aplastamos no reviven". ${ }^{29}$ Desafortunadamente, la cita parece continuar más que vigente en el debate pedagógico y educativo, tanto desde perspectivas prácticas como teóricas.

La premisa original del pensamiento ético de Dewey tiene en su base la interacción y la comunicación, pues su ética nace de la observación de que el ser humano es un animal social, y como tal desea comunicarse. En su apuesta por una escuela democrática, el pensador y pedagogo defendía que la escuela no ha de preparar para la vida, pues ya es la vida. La escuela, entendida como laboratorio filosófico y democrático para el presente, adquiere por consiguiente un enfoque funcional, y no educativo preventivo o futurible. Esta idea, pilar de los enfoques pedagógicos y didácticos funcionales, se fundamenta en que el niño encuentre significación y sentido (función) a los aprendizajes. De este modo, y por poner un ejemplo, el escolar no leerá en sus primeros años para aprender a leer, sino para saber lo que hay escrito. Como respuesta a su talante democrático, el propósito general de toda tarea pedagógica se traducirá en el respeto por las diferencias de necesidades, intereses y ritmos de aprendizaje.

En la visión de la diferencia, la vulnerabilidad adquiere un papel esencial, pues se fundamenta en una narrativa a caballo entre el engaño y el rechazo de lo disímil. Es ilusoria, pues tratamos de manejar nuestra inseguridad ontológica en una "fícción de invulnerabilidad" 30 que haga la existencia más soportable. Es rechazada, en tanto en cuanto la negamos para no enfrentarnos a su consustancialidad a lo humano. Entender al vulnerable (y su condición) como alejado de la propia esencia da lugar, pues, a una mentira ontológica.

Todo otro es, por definición, una alteración a cualquier idea de normalidad, dirá Skliar. ${ }^{31}$ Tal alteración pone en jaque la dicotomía entre lo normal y lo anormal. La raridad, dirán Coca y Rodríguez, no es sino la baja prevalencia de un suceso, y suele traer

\footnotetext{
${ }^{29}$ John Dewey, Democracy and education (Nueva York: Mcmillan, 1916), cit. en Noddings, La educación moral, 69-70.

${ }^{30}$ Martha C. Nussbaum. El ocultamiento de lo humano: repugnancia, vergüenza y ley (Buenos Aires: Katz Editores, 2006).

${ }^{31}$ Carlos Skliar, Pedagogía de las diferencias. Notas, fragmentos e incertidumbres (Buenos Aires: Noveduc / Graó, 2017).
} 
de la mano a la indeseabilidad, el rechazo y la culpa. ${ }^{32}$ El raro, por defecto o exceso de mirada, es arrojado al terreno de la invisibilidad o de la sobreexposición. Sea una u otra la mirada socioeducativa, esta derivará en exclusión, bien por exclusión directa o por lo que se ha llamado una inclusión excluyente.

Entender al vulnerable, al diferente, como raro y alejado de uno y de los que supuestamente son más semejantes en la gradación de analogías, le destierra a la periferia, o incluso a la negación, de la semiosis relacional y socioeducativa. En esto radica la exclusión: en situar al otro en un lugar en el que no hay interacción posible.

Ver, así, al otro como signo aislado en su diferencia, lo catapulta a los márgenes y lo hace más vulnerable, pues le niega la posibilidad de ser signo entre signos y generar nuevas (y comunes) significaciones y sentidos. El signo-persona queda exiliado de la comunidad y los procesos sociales como contextos humanos y humanizadores.

Pero la diferencia nunca puede ser sujeto, sino relación, ${ }^{33}$ pues sólo en y desde la interacción es posible vislumbrar distinciones o semejanza. Necesitamos del otro para saber nuestra medida, dirá la hermenéutica analógica, un salir de sí para que el signo-persona pueda encontrar al diferente y, ya en la tarea pedagógica, intuir lo que necesita saber. La raridad entendida como aislamiento impide toda novedad, todo hallazgo, pues en la ausencia de relación elimina al interpretante, y con él la posibilidad de crear nuevos signos. Es la negación de toda semiosis.

"La relación de alteridad no expresa ninguna 'necesidad' del sujeto porque si no fuese así, la relación con el otro sería una relación interesada". ${ }^{34}$ El homo pedagógico está diseñado para afrontar la responsabilidad de sostener y poner en común. Desde esta perspectiva, el educador llevará en su código genético la vocación de la ética y la alteridad.

Para Pié Balaguer, es esta interdependencia donde estriba la condición de nuestra propia autonomía, esto es, la posibilidad de ser. Por su parte, Ruddick señala al sostenimiento como un modo de ver las cosas (un modo, podría decirse, de estar en el

\footnotetext{
32 Juan R. Coca y Juan Antonio Rodríguez Sánchez, "Transformaciones en las fronteras socio-biomédicas: análisis teórico sobre las Enfermedades Raras", en Juan Antonio Roche Cárcel (Ed.), Las sociedades difusas La construcción/deconstrucción sociocultural de fronteras y márgenes. (Barcelona: Anthropos, 2020).

33 Skliar, Pedagogía de las diferencias.

${ }^{34}$ Joan Carles Mèlich, La ausencia del testimonio: Ética y pedagogía en los relatos del Holocausto (Barcelona: Anthropos, 2001), 66-67.
} 
mundo y ser signo entre signos) cuyas miras son conservar la armonía, los recursos y las destrezas para que el niño se sienta seguro. Esto requiere de la creación de contextos socioeducativos amables, responsables y de cuidado (en los que, en línea con la ética de Noddings, se aprenda a cuidar a partir de la experiencia de ser cuidado), así como de continuidad y de atención.

Es el respeto, o la mirada atenta, que Esquirol ${ }^{35}$ propone: “detenernos y atender". Es recibir al otro, desde una pedagogía de la hospitalidad ${ }^{36}$ o de las diferencias, y que esta pedagogía sea, en términos de Van Manen la fascinación por el crecimiento del otro. Y crecer, ya lo hemos señalado con Peirce, no es tan solo aumentarse como signos, sino relacionarse, salir de sí, establecer procesos edusemióticos creativos, novedosos, responsables y atentos a la interdependencia, la alteridad y, cómo no, a la vulnerabilidad de lo humano.

\footnotetext{
${ }^{35}$ Josep M. Esquirol, El respeto o la mirada atenta. Una ética para la era de la ciencia y la tecnología (Barcelona: Gedisa, 2006).

${ }^{36}$ Fernando Bárcena y Joan Carles Mèlich, La educación como acontecimiento ético. Natalidad, narración y hospitalidad (nueva edición revisada y aumentada) (Buenos Aires: Miño y Dávila, 2014).
} 


\section{Bibliografía}

Arendt, Hannah. "La crisis de la educación". En Entre el pasado y el futuro. Ocho ejercicios sobre la reflexión política (185-208). Barcelona: Ediciones Península, 1996.

Barrena, Sara, y Jaime Nubiola. Charles S Peirce (1839-1914): Un pensador para el siglo XXI. Navarra: Ediciones Universidad de Navarra, 2013.

Beuchot, Mauricio. Tratado de hermenéutica analógica: hacia un nuevo modelo de interpretación. Ciudad de México: Universidad Autónoma Metropolitana, 2000.

Beuchot, Mauricio. Hermenéutica, analogía y símbolo. Barcelona: Herder, 2004.

Beuchot, Mauricio. Perfiles esenciales de la hermenéutica. Ciudad de México: Fondo de Cultura Económica, 2008.

Castoriadis, Cornelius. La institución imaginaria de la sociedad. Barcelona: Tusquets, 2013.

Coca, Juan R., y Juan Antonio Rodríguez Sánchez. "Transformaciones en las fronteras socio-biomédicas: análisis teórico sobre las Enfermedades Raras”. En Juan Antonio Roche Cárcel (Ed.), Las sociedades difusas La construcción/deconstrucción sociocultural de fronteras y márgenes. Barcelona: Anthropos, 2020.

Danesi, Marcel. "Foreword: Edusemiotics". En Inna Semetsky (ed.), Edusemiotics. A handbook. Singapore: Springer, 2010.

Esquirol, Josep M. El respeto o la mirada atenta. Una ética para la era de la ciencia y la tecnología. Barcelona: Gedisa, 2006.

Mèlich, Joan Carles. La ausencia del testimonio: Ética y pedagogía en los relatos del Holocausto. Barcelona: Anthropos, 2001.

Noddings, Nel. La educación moral. Propuesta alternativa para la educación del carácter. Buenos Aires: Amorrortu, 2009.

Nussbaum, Martha C. El ocultamiento de lo humano: repugnancia, vergüenza y ley. Buenos Aires, Madrid: Katz Editores, 2006.

Kondrátov, Aleksandr Mikhaūlovich. Del Sonido al Signo. Buenos Aires: Paidós, 1973.

Piaget, Jean. Introduccción a la psicolingüística. Buenos Aires: Nueva Visión, 1969.

Piaget, Jean. El lenguaje y el pensamiento del niño pequeño. Barcelona: Paidós, 1984.

Piaget, Jean. Inteligencia y afectividad. Buenos Aires: Aique, 2001. 
Piaget, Jean. El nacimiento de la inteligencia en el niño. Barcelona: Editorial Crítica, 2014.

Peirce, Charles Sanders. Fundamento, objeto e interpretante (2.228, c. 1897). Texto tomado de MS 798 [On Signs] c.1897, Publicado como Collected Papers (CP) 2.227-229 y $2.444 \mathrm{n} 1,2003$.

Peirce, Charles Sanders. Collected Papers, vol. 1-8, 1931-1958. C. Hartshorne, P. Weiss y A. W. Burks (eds.). Cambridge, MA: Harvard University Press.

Pié Balaguer, Asun. La insurrección de la vunerabilidad. Para una pedagogía de los cuidados y la resistencia. Barcelona: Edicions Universitat Barcelona, 2019.

Pozo, Juan Ignacio. "La nueva cultura del aprendizaje en la sociedad del conocimiento". En Juan Ignacio Pozo, Nora Scheuer, María del Puy Pérez Echeverria, María del Mar Mateos, Elena Martín y Montserrat de La Cruz (Eds.), Nuevas formas de pensar la enseñanza y el aprendizaje: las concepciones de profesores y alumnos. Barcelona: Graó, 2015.

Pozo, Juan Ignacio. Adquisición del conocimientok. Madrid: Morata, 2003.

Savater, Fernando. "La educación es el momento adecuado de la ética. ¿De qué sirve la ética para los jóvenes?”. Educere 4, núm. 11 (2000): 215-226

Touriñán, José Manuel. "Donde hay educación, hay riesgo: además de enseñar, hay que educar”. Voces de la Educación 1, núm. 1 (2016): 115.

Semetsky, Inna (Ed.). Semiotics Education Experience. Rotterdam: Sense Publishers, 2010.

Semetsky, Inna. "Introduction: A Primer on Edusemiotics". En Inna semetsky (Ed.), Edusemiotics. A Handbook. Singapore: Springer, 2017.

Schleiermaher, Friedrich. Hermeneutics and criticism and other writings. Cambridge: Cambridge University Press, 1989.

Skliar, Carlos. Pedagogía de las diferencias. Notas, fragmentos e incertidumbres. Buenos Aires: Noveduc / Graó, 2017.

Van Manen, Max. El tacto en la enseñanza. El significado de la sensibilidad pedagógica. Barcelona: Paidós Educador, 2018.

Vigotsky, Lev Semionovich. Pensamiento y lenguaje. Barcelona: Paidós Ibérica, 2020. 